\title{
Temperature-Dependent Mollow Triplet Spectra from a Single Quantum Dot: Rabi Frequency Renormalization and Sideband Linewidth Insensitivity
}

Wei, Yu-Jia; He, Yu; He, Yu-Ming; Lu, Chao-Yang; Pan, Jian-Wei; Schneider, Christian; Kamp, Martin; Höfling, Sven; McCutcheon, Dara

Published in:

Physical Review Letters

Link to article, DOI:

10.1103/PhysRevLett.113.097401

Publication date:

2014

Document Version

Publisher's PDF, also known as Version of record

Link back to DTU Orbit

Citation $(A P A)$ :

Wei, Y-J., He, Y., He, Y-M., Lu, C-Y., Pan, J-W., Schneider, C., Kamp, M., Höfling, S., \& McCutcheon, D. (2014). Temperature-Dependent Mollow Triplet Spectra from a Single Quantum Dot: Rabi Frequency Renormalization and Sideband Linewidth Insensitivity. Physical Review Letters, 113, [097401-1]. https://doi.org/10.1103/PhysRevLett.113.097401

\section{General rights}

Copyright and moral rights for the publications made accessible in the public portal are retained by the authors and/or other copyright owners and it is a condition of accessing publications that users recognise and abide by the legal requirements associated with these rights.

- Users may download and print one copy of any publication from the public portal for the purpose of private study or research.

- You may not further distribute the material or use it for any profit-making activity or commercial gain

- You may freely distribute the URL identifying the publication in the public portal 


\title{
Temperature-Dependent Mollow Triplet Spectra from a Single Quantum Dot: Rabi Frequency Renormalization and Sideband Linewidth Insensitivity
}

\author{
Yu-Jia Wei, Yu He, Yu-Ming He, Chao-Yang Lu, ${ }^{\ddagger}$ and Jian-Wei Pan ${ }^{\S}$ \\ Hefei National Laboratory for Physical Sciences at the Microscale and Department of Modern Physics, \\ \& CAS Center for Excellence and Synergetic Innovation Center in Quantum Information and Quantum Physics, \\ University of Science and Technology of China, Hefei, Anhui 230026, China \\ Christian Schneider and Martin Kamp \\ Technische Physik, Physikalisches Institut and Wilhelm Conrad Röntgen-Center for Complex Material Systems, \\ Universität Würzburg, Am Hubland, D-97074 Würzburg, Germany
}

Sven Höfling

SUPA, School of Physics and Astronomy, University of St. Andrews, Saint Andrews KY16 9SS, United Kingdom; Technische Physik, Physikalisches Institut and Wilhelm Conrad Röntgen-Center for Complex Material Systems, Universität Würzburg, Am Hubland, D-97074 Würzburg, Germany; and Hefei National Laboratory for Physical Sciences at the Microscale and Department of Modern Physics, University of Science and Technology of China, Hefei, Anhui 230026, China

Dara P. S. McCutcheon"*

Department of Photonics Engineering, DTU Fotonik, Ørsteds Plads, 2800 Kgs Lyngby, Denmark and Departamento de Física, FCEyN, UBA and IFIBA, Conicet, Pabellón 1, Ciudad Universitaria, 1428 Buenos Aires, Argentina

\begin{abstract}
Ahsan Nazir ${ }^{\dagger}$
Photon Science Institute \& School of Physics and Astronomy, University of Manchester, Oxford Road, Manchester M13 9PL, United Kingdom and Controlled Quantum Dynamics Theory, Imperial College London, London SW7 2AZ, United Kingdom (Received 20 December 2013; revised manuscript received 18 June 2014; published 28 August 2014)
\end{abstract}

\begin{abstract}
We investigate temperature-dependent resonance fluorescence spectra obtained from a single selfassembled quantum dot. A decrease of the Mollow triplet sideband splitting is observed with increasing temperature, an effect we attribute to a phonon-induced renormalization of the driven dot Rabi frequency. We also present first evidence for a nonperturbative regime of phonon coupling, in which the expected linear increase in sideband linewidth as a function of temperature is canceled by the corresponding reduction in Rabi frequency. These results indicate that dephasing in semiconductor quantum dots may be less sensitive to changes in temperature than expected from a standard weak-coupling analysis of phonon effects.
\end{abstract}

DOI: 10.1103/PhysRevLett.113.097401

PACS numbers: 78.67.Hc, 71.38.-k, 78.47.-p

Self-assembled semiconductor quantum dots (QDs) provide a promising platform for quantum information processing using single spins [1,2] and photons [3-6]. Such applications require QD quantum coherence to be preserved on time scales sufficient for performing high fidelity quantum operations, and for emitted single photons to possess a large degree of indistinguishability [7-9]. Indistinguishable photons can be produced by $s$-shell resonant optical excitation of a single QD [10-12], wherein an electron-hole pair is created directly without any relaxation from higher states, which would otherwise cause inhomogeneous broadening in the QD emission spectrum. The coherence time $\left(T_{2}\right)$ of such photons is able to approach the Fourier transform limit, $T_{2}=2 T_{1}$ (with $T_{1}$ the QD radiative lifetime) at low temperatures $(\sim 4 \mathrm{~K})$ and weak driving strengths [13].

As the intensity of the pump laser increases, however, an additional power dependent dephasing contribution arises, even at low temperatures [14-24]. This is often termed excitation induced dephasing (EID), which commonly originates from deformation potential coupling of QD excitons to longitudinal acoustic (LA) phonons. As the driving strength increases, so does the energy splitting of the excitonic dressed states, and excitations are then able to scatter with the increased density of phonons around this energy scale in the bulk semiconductor lattice. Driving dependence is thus a pronounced characteristic of EID, as was observed in Refs. [14,15], where QD excitonic Rabi oscillations were measured via photocurrents, and in Refs. [16,17] through driven QD optical emission.

Besides EID, another direct manifestation of the phonon influence on a driven dot can be found in the dependence of its properties on temperature. In fact, an idealized two-level system (e.g., an isolated atom) should not show any change in emission behavior with temperature over the usual experimental range, in contrast to the response to changes 
in driving strength, which is nontrivial even in the absence of phonons [25]. Though the damping of excitonic Rabi rotations (in the QD population) has been measured at various temperatures under pulsed excitation [14,15,17], the direct observation of temperature dependence in the field correlation properties and resonance fluorescence (RF) emission spectra of a single QD has not been reported. This is arguably the most relevant scenario for photonic applications, since it is crucial to understanding the competing roles played by the electromagnetic and solid-state environments in determining the QD optical emission characteristics and coherence [26-29].

Here, we present a combined experimental and theoretical investigation of temperature dependence in the RF spectra obtained from a single self-assembled QD, generated by continuous-wave (cw) $s$-shell excitation. We show that the coupling of the QD neutral exciton to LA phonons leads to a temperature-dependent phonon renormalization (suppression) of the driven dot Rabi frequency, which we can observe directly through a decrease in Mollow triplet sideband splitting with increasing temperature. This trend is in agreement with an exciton-phonon coupling model based on a polaron master equation technique $[23,26-28]$. Our theoretical analysis also predicts a nonperturbative regime of phonon coupling, wherein a linear increase of the sideband linewidths with temperature (as would be found from a standard weak-coupling analysis [14]) is appreciably canceled by the corresponding decrease in the phononrenormalized Rabi frequency [23]. This prediction is supported by our experimental data, demonstrating an experimental regime in which EID is less sensitive to changes in temperature than would be expected from a perturbative, weak exciton-phonon coupling model [14,15,19,22].

Our experiments are performed on a single selfassembled InAs/GaAs QD embedded in the center of a microcavity with a low $Q$ factor $(\sim 200)$ in order to enhance fluorescence collection efficiency. The sample is kept in a cryogen-free bath cryostat with the temperature stabilized within $\pm 5 \mathrm{mK}$ around a fixed value, as measured by a thermometer on the sample holder [30]. A cw pump is provided by a semiconductor diode laser, with frequency stabilized to within $\pm 2 \pi \times 2 \mathrm{MHz}$, which is much narrower than the linewidth of the QD fluorescence. Figure 1 shows the cross-polarization setup used to achieve resonant excitation [13]. The neutral single exciton of the QD has two fine-structure states, whose dipole moment orientations we label $\mathbf{X}$ and $\mathbf{Y}$. As seen in Fig. 1(c), these states are split by approximately $2 \mathrm{GHz}$. We resonantly excite the $\mathbf{Y}$ transition with a polarization approximately orthogonal to the collection polarization. Since $\mathbf{X}$ and $\mathbf{Y}$ are not strictly orthogonal, and the $\mathbf{X}$ transition is detuned and orthogonal to the collection polarization $\mathbf{C}$, we can selectively measure $\mathrm{RF}$ emission from the $\mathbf{Y}$ transition while extinguishing the pump laser with a ratio exceeding $10^{6}$. Nonorthogonality of the fine structure states may be due to imperfect selection

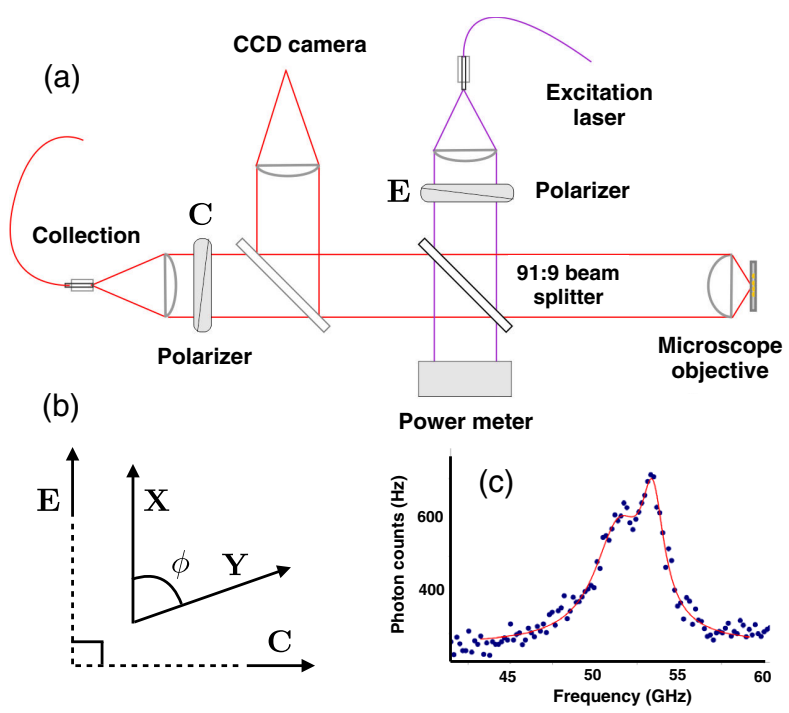

FIG. 1 (color online). The cross-polarization experimental setup is shown in (a), where vectors associated with the polarizers in the excitation $\mathbf{E}$, and collection $\mathbf{C}$ arms are approximately orthogonal, as shown in (b). Also shown schematically in (b) are the dipole moment orientation vectors of the two fine-structure split states $\mathbf{X}$ and $\mathbf{Y}$. These states are split by approximately $2 \mathrm{GHz}$ as confirmed by above band photoluminescence measurements shown in (c).

rules within the solid state, and has been reported elsewhere [31]. Finally, a Fabry-Perot interferometer is used to resolve the RF spectra with a spectral resolution of $\sim 2 \pi \times 67 \mathrm{MHz}$ (which we account for in our fittings below).

We begin by analyzing QD RF spectra at fixed temperature (4 K), for a series of cw laser driving strengths [5]. This serves as a form of calibration of our system, and through a comparison with theory provides us with necessary parameters for our subsequent examination of temperature changes in the spectra. High resolution RF spectra from the neutral exciton state were obtained for various laser powers [5]. For increasing driving strength, the expected triple peak structure in the RF spectra, known as the Mollow triplet [25], was observed. This can be interpreted in the dressed state picture [32], in which the QD exciton is dressed through its interaction with the driving laser. This causes the originally degenerate, uncoupled QD-photon states to split into two states separated by $\Omega_{r}$, where $\Omega_{r}$ is the effective phonon-renormalized strength of the QD-laser interaction. Photons can then be emitted corresponding to four different transitions, two at the QD transition frequency, and two more offset by $\pm \Omega_{r}$. In the absence of phonons, and within the dipole approximation, $\Omega_{r} \rightarrow \Omega=\mu E / \hbar$ is just the bare Rabi frequency, where $\mu$ is the effective dot dipole moment and $E$ is the amplitude of optical field at the QD.

The presence of phonons, however, introduces both additional broadening and a renormalization of the effective Rabi strength, $\Omega \rightarrow \Omega_{r}$. To account for these processes, we 
exploit a polaron master equation technique [23,26-28], which allows us to derive an explicit expression for the emission spectrum of the QD in terms of experimental parameters and microscopic constants characterizing the QD-phonon coupling. We model the excitonic degree of freedom of the QD as a two-level-system, and couple it to two harmonic oscillator baths, which represent both the electromagnetic field into which light is emitted, and the phonon environment present in the substrate. In Ref. [28] it was shown that provided $\Omega<k_{B} T<\omega_{c}$, with $T$ the temperature and $\omega_{c}$ the cutoff frequency of the phonon environment (related to the size of the QD [15]), the influence of phonons on the incoherent emission spectrum can be captured entirely by a renormalization of the bare Rabi frequency and the introduction of a pure-dephasing rate. Above saturation, where $\Omega_{r} \gg \Gamma_{1}$, with $\Gamma_{1}=1 / T_{1}$ and $T_{1}=390 \pm 10$ ps determined by time resolved correlation measurements, the emission spectrum is given by $S(\omega) \propto \operatorname{Re}\left[\int_{0}^{\infty} d \tau e^{i\left(\omega-\omega_{l}\right) \tau} g_{1}(\tau)\right]$, in terms of the first order field correlation function

$$
g_{1}(\tau) \approx \frac{1}{4}\left(e^{-\Gamma_{2} \tau}+e^{-(1 / 2)\left(\Gamma_{1}+\Gamma_{2}\right) \tau} \cos \left(\Omega_{r} \tau\right)\right),
$$

with $\omega_{l}$ the frequency of the excitation laser. The renormalized Rabi frequency is (we set $\hbar=1$ )

$$
\Omega_{r}(T)=\Omega \exp \left[-\frac{1}{2} \int_{0}^{\infty} d \omega \frac{J(\omega)}{\omega^{2}} \operatorname{coth}\left(\frac{\omega}{2 k_{B} T}\right)\right],
$$

where $J(\omega)$ is the spectral density of the QD-phonon interaction, which for coupling to LA phonons has been shown to be adequately described by the functional form $J(\omega)=\alpha \omega^{3} \exp \left[-\left(\omega / \omega_{c}\right)^{2}\right]$, with $\alpha$ capturing the strength of the interaction $[14,15]$. The phonon-induced puredephasing rate enters through $\Gamma_{2}=(1 / 2) \Gamma_{1}+\gamma_{\mathrm{PD}}+\gamma_{0}$, and is given by

$$
\gamma_{\mathrm{PD}}=\left(\frac{\Omega_{r}}{2}\right)^{2} \int_{-\infty}^{\infty} d s \cos \left(\Omega_{r} s\right)\left(e^{\phi(s)}-e^{-\phi(s)}\right),
$$

with $\phi(s)=\int_{0}^{\infty} d \omega J(\omega) \omega^{-2}\left[\cos (\omega s) \operatorname{coth}\left(\omega / 2 k_{B} T\right)-\right.$ $i \sin (\omega s)]$. We also include a term $\gamma_{0}$ to account for any additional driving-independent pure-dephasing processes.

In the regime described above, the spectrum is given simply by the sum of three Lorentzians corresponding to the three peaks in the Mollow triplet. We are interested here in the sidebands, which have positions at $\pm \Omega_{r}$ and a full width at half maximum (FWHM) of

$$
\Delta \omega=\Gamma_{1}+\Gamma_{2}=\frac{3}{2} \Gamma_{1}+\gamma_{\mathrm{PD}}+\gamma_{0} .
$$

By expanding $\gamma_{\mathrm{PD}}$ in a single-phonon limit, i.e., $e^{ \pm \phi(s)} \approx$ $1 \pm \phi(s)$ in Eq. (3), the phonon-induced pure-dephasing rate can be approximated by $\gamma_{\mathrm{PD}} \approx(\pi / 2) J\left(\Omega_{r}\right) \operatorname{coth}\left(\Omega_{r} / 2 k_{B} T\right)$ [23], which we can further simplify to

$$
\gamma_{\mathrm{PD}}\left(\Omega_{r}, T\right) \approx \pi \alpha k_{B} T \Omega_{r}^{2},
$$

provided that $\Omega_{r} \ll k_{B} T, \omega_{c}$ [15,23]. Under these constraints, which are satisfied in our experiments, the FWHM of the sidebands varies linearly with $\Omega_{r}^{2}$, with a gradient proportional to the exciton-phonon coupling constant $\alpha$. Despite the single-phonon expansion, Eq. (5) still captures important nonperturbative phonon effects through the renormalized $\Omega_{r}$, which is itself dependent (nonlinearly) upon the coupling strength $\alpha$ and the temperature $T$ [see Eq. (2)]. The phonon-induced dephasing rate given by Eq. (5) is thus distinct from that obtained via a secondorder weak-coupling treatment of phonon interactions, in which $\Omega_{r}$ would be replaced by the bare, unrenormalized $\Omega$ $[14,15]$, which is independent of $\alpha$ or $T$. This will be particularly important in describing the temperature dependent behavior we observe below.

To obtain Fig. 2 we fit each spectrum to a sum of three Lorentzians to determine experimental values for the positions and widths of the side peaks. Figure 2(a) shows the extracted renormalized driving strength as a function of the square root of the laser power, $\sqrt{P} \sim E$, displaying a linear dependence as expected. In Fig. 2(b) we plot the extracted sideband widths of the blue (centered at $\Omega_{r}$ ) and red (centered at $-\Omega_{r}$ ) sidebands as a function of $\Omega_{r}^{2}$, also showing the anticipated linear behavior. Using the FWHM expression given in Eq. (4), we obtain values of $\alpha=$ $(2.535 \pm 0.156) \times 10^{-7} \mathrm{GHz}^{-2}$ and $\gamma_{0}=0.135 \pm 0.062 \mathrm{GHz}$ from the data in Fig. 2(b). The nonzero value of $\gamma_{0}$ implies that $\Gamma_{1} / 2 \Gamma_{2} \approx 0.9$ at vanishing driving strength $\left(\Gamma_{1} / 2 \Gamma_{2}=1\right.$ in the Fourier transform limit) and may possibly be due to fluctuating charges trapping in the vicinity of the QD [33-35].

We now turn our attention to the dependence on temperature in the RF spectra. This was measured in the range of 4-14 K, with the nominal laser power fixed at $9.9 \mu \mathrm{W}$, as shown in Fig. 3(a). The purple markers in Fig. 3(b) show the extracted effective Rabi frequency $\Omega_{r}$ as a function of temperature. The data reveal a clear decrease of $\Omega_{r}$ as the
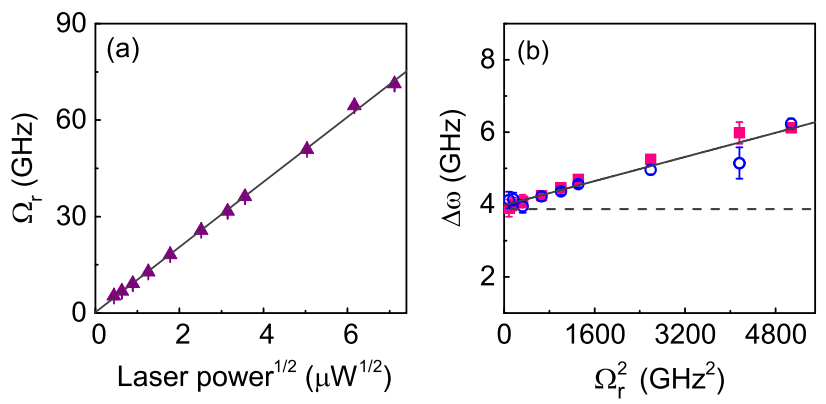

FIG. 2 (color online). (a) Extracted phonon renormalized Rabi frequency $\Omega_{r}$ as a function of the square root of the cw laser power $\sqrt{P}$, together with a linear fit (gray solid line). (b) Extracted linewidths of the red (red filled squares) and blue (blue open circles) sidebands as a function of $\Omega_{r}^{2}$. A straight line fit to the data points is shown in gray (solid line). The dashed line shows the expected linewidth in the absence of any pure dephasing (driving dependent or otherwise), i.e., the Fourier transform limit. 

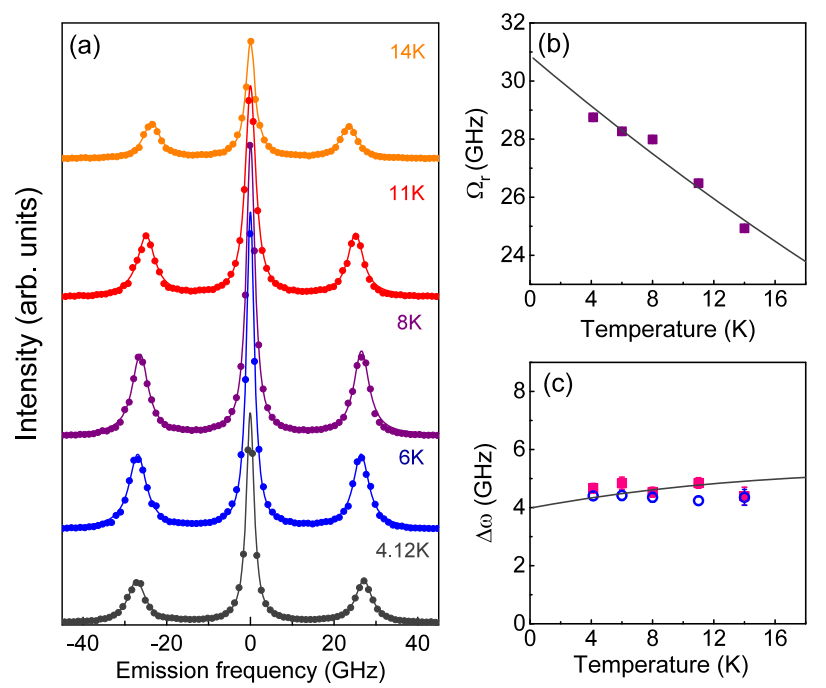

FIG. 3 (color online). (a) High resolution RF spectra of the neutral exciton emission at different temperatures. The temperature spans $4-14 \mathrm{~K}$, and the nominal cw laser power is $9.9 \mu \mathrm{W}$. (b) Phonon-renormalized Rabi frequency $\Omega_{r}$ as a function of temperature. The data are fitted to Eq. (2), shown as the solid curve. (c) Extracted linewidths of the red (red filled squares) and blue (blue open circles) sidebands as a function of temperature. The solid curve shows the expected trend from our nonperturbative exciton-phonon coupling theory, using parameters already determined (i.e., it is not a fit to the data).

temperature is increased. This is the most striking evidence of coupling to phonons in our experiment, since the Rabi frequency of a driven atom in the traditional setting of quantum optics is determined only by the value of the dipole moment and the optical field amplitude, which are both constant in these measurements.

The renormalization of the Rabi frequency is entirely consistent with our exciton-phonon coupling model. As previously stated, in the regime in which we are working, the positions of the Mollow sidebands are given approximately by $\pm \Omega_{r}$, as defined in Eq. (2). From the theory, we expect $\Omega_{r}$ to decrease with temperature for a fixed driving power, with a slope determined by the spectral density parameters $\alpha$ and $\omega_{c}$, which together characterize the overall strength of the QD-phonon interaction. Using the extracted value of $\alpha=2.535 \times 10^{-7} \mathrm{GHz}^{-2}$ from the driving dependent data, we fit the experimentally measured values of $\Omega_{r}$ to Eq. (2) to find a bare Rabi frequency of $\Omega=30.88 \pm 0.52 \mathrm{GHz}$, and a spectral density cutoff frequency of $\omega_{c}=493.33 \pm 61.28 \mathrm{GHz}$. We note that this implies $\omega_{c} \gg \Omega_{r}$ for all driving strengths considered previously, thus confirming our assumption that $\exp \left[-\left(\Omega_{r} / \omega_{c}\right)^{2}\right] \approx 1$ used to obtain Eq. (5).

To complete the picture we now consider how the sideband widths vary with temperature. The data points plotted in Fig. 3(c) show the extracted widths of the blue and red sidebands, while the solid curve shows the expected trend from our theoretical analysis, for which the FWHM is given by Eq. (4) using $\gamma_{\mathrm{PD}}$ as defined in Eq. (5). We note that having fully determined all parameters from other measurements, there is no fitting procedure used to obtain this curve. Intriguingly, we see that theoretically we expect a sublinear increase in the sideband widths across this temperature range, which also appears to be supported by the data. From Eq. (5), the phonon-induced dephasing rate depends on the product $\alpha T \Omega_{r}^{2}$. The explicit dependence on $T$ is a first order process, reflecting the increase in the occupation number of phonons with a frequency corresponding to the Rabi energy. The implicit dependence on $T$ through $\Omega_{r}$, however, is a nonperturbative effect describing the renormalization of the dressed state energy splitting which serves to reduce the dephasing rate. A competition between these two processes gives rise to an overall weak dependence on temperature for the parameters we have determined here.

We stress again that this effect cannot be captured by a perturbative weak-coupling treatment, in which $\Omega_{r} \rightarrow \Omega$ independent of temperature. In such a case the dephasing rate, and hence the sideband widths, would increase linearly with temperature $[14,15,17,23]$. However, it is important to note that the nonlinear temperature dependence we observe is not at odds with the linear dependence seen in Refs. [14,15,17]. The difference in behavior arises as a result of the nonperturbative regime in which our experimentally determined parameters place us, and thus depends sensitively on the phonon coupling characteristics of the individual QD sample studied. Specifically, we extract a larger value of the coupling strength $\alpha$ than in Refs. [14,15,17] and also a smaller cutoff frequency $\omega_{c}$, the latter of which suggests a larger QD size due to the inverse relationship between the two [14,15]. The combination of a larger $\alpha$ and smaller $\omega_{c}$ ensures that the renormalization of the Rabi frequency, which ultimately gives rise to the nonlinear temperature dependence [see Eq. (2)], is enhanced in our experiments.

In summary, we have presented the first systematic exploration of temperature dependence in the RF emission spectra of a solid-state QD emitter. Our experiments and theoretical description were first calibrated through an analysis of the dependence on driving strength, in which we confirmed the expected increase in sideband splitting and linewidth with increasing driving strength. We then showed a systematic decrease of sideband splitting with increasing temperature at constant driving, an effect we attribute to a phonon-induced temperature-dependent suppression of the effective Rabi frequency $\Omega_{r}$ [23]. Resulting from this renormalization, we observed evidence of nonperturbative phonon coupling effects in the insensitivity of sideband linewidths to an increase in temperature. This suggests that changes in temperature may not be as detrimental to QD coherence as a weak-coupling analysis would otherwise indicate, which could have important repercussions in the application of QDs to future quantum technologies-such as high quality single photon generation in strong cavity-QD coupling systems [36] and 
QD-based thermometry [37] —as well as for other solidstate emitters [38-40].

We thank C. M. Chen and Z. G. Lu for helpful discussions. This work was supported by the National Natural Science Foundation of China, the Chinese Academy of Sciences, the National Fundamental Research Program (under Grants No. 2011CB921300 and No. 2013CB933300), the State of Bavaria, by the BMBF and EPSRC within the CHIST-ERA project SSQN, and project SIQUTE (Contract No. EXL02) of the European Metrology Research Programme (EMRP). The EMRP is jointly funded by the EMRP participating countries within EURAMET and the European Union. S. H. acknowledges the CAS visiting professorship. D. P.S. M. thanks CONICET and A. N. thanks Imperial College London and the University of Manchester for financial support.

*daramc@fotonik.dtu.dk

†ahsan.nazir@manchester.ac.uk

¥cylu@ustc.edu.cn

§pan@ustc.edu.cn

[1] R. J. Warburton, Nat. Mater. 12, 483 (2013).

[2] S. Benjamin, B. Lovett, and J. M. Smith, Laser Photonics Rev. 3, 556 (2009).

[3] P. Michler et al., Science 290, 2282 (2000).

[4] C. Santori, D. Fattal, J. Vučković, G. S. Solomon, and Y. Yamamoto, Nature (London) 419, 594 (2002).

[5] Y.-M. He, Y. He, Y.-J. Wei, D. Wu, M. Atatüre, C. Schneider, S. Höfling, M. Kamp, C.-Y. Lu, and J.-W. Pan, Nat. Nanotechnol. 8, 213 (2013).

[6] A. Dousse, J. Suffczyński, A. Beveratos, O. Krebs, A. Lemaître, I. Sagnes, J. Bloch, P. Voisin, and P. Senellart, Nature (London) 466, 217 (2010).

[7] E. B. Flagg, A. Muller, S. V. Polyakov, A. Ling, A. Migdall, and G. S. Solomon, Phys. Rev. Lett. 104, 137401 (2010).

[8] R. B. Patel, A. J. Bennett, I. Farrer, C. A. Nicoll, D. A. Ritchie, and A. J. Shields, Nat. Photonics 4, 632 (2010).

[9] A. Nazir and S. D. Barrett, Phys. Rev. A 79, 011804(R) (2009).

[10] A. Müller, E. B. Flagg, P. Bianucci, X. Y. Wang, D. G. Deppe, W. Ma, J. Zhang, G. J. Salamo, M. Xiao, and C. K. Shih, Phys. Rev. Lett. 99, 187402 (2007).

[11] A. N. Vamivakas, Y. Zhao, C.-Y. Lu, and M. Atatüre, Nat. Phys. 5, 198 (2009).

[12] E. B. Flagg, A. Muller, J. W. Robertson, S. Founta, D. G. Deppe, M. Xiao, W. Ma, G. J. Salamo, and C. K. Shih, Nat. Phys. 5, 203 (2009).

[13] C. Matthiesen, A. N. Vamivakas, and M. Atature, Phys. Rev. Lett. 108, 093602 (2012).

[14] A. J. Ramsay, A. Venu Gopal, E. M. Gauger, A. Nazir, B. W. Lovett, A. M. Fox, and M. S. Skolnick, Phys. Rev. Lett. 104, 017402 (2010).

[15] A. J. Ramsay, T. M. Godden, S. J. Boyle, E. M. Gauger, A. Nazir, B. W. Lovett, A. M. Fox, and M. S. Skolnick, Phys. Rev. Lett. 105, 177402 (2010).

[16] S. M. Ulrich, S. Ates, S. Reitzenstein, A. Löffler, A. Forchel, and P. Michler, Phys. Rev. Lett. 106, 247402 (2011).
[17] L. Monniello, C. Tonin, R. Hostein, A. Lemaitre, A. Martinez, V. Voliotis, and R. Grousson, Phys. Rev. Lett. 111, 026403 (2013).

[18] J. Förstner, C. Weber, J. Danckwerts, and A. Knorr, Phys. Rev. Lett. 91, 127401 (2003).

[19] P. Machnikowski and L. Jacak, Phys. Rev. B 69, 193302 (2004).

[20] A. Vagov, M. D. Croitoru, V. M. Axt, T. Kuhn, and F. M. Peeters, Phys. Rev. Lett. 98, 227403 (2007).

[21] D. Mogilevtsev, A. P. Nisovtsev, S. Kilin, S. B. Cavalcanti, H. S. Brandi, and L. E. Oliveira, Phys. Rev. Lett. 100, 017401 (2008).

[22] A. Nazir, Phys. Rev. B 78, 153309 (2008).

[23] D. P. S. McCutcheon and A. Nazir, New J. Phys. 12, 113042 (2010).

[24] H. Kim, T. C. Shen, K. Roy-Choudhury, G. S. Solomon, and E. Waks, Phys. Rev. Lett. 113, 027403 (2014).

[25] B. R. Mollow, Phys. Rev. 188, 1969 (1969).

[26] C. Roy and S. Hughes, Phys. Rev. Lett. 106, 247403 (2011).

[27] C. Roy and S. Hughes, Phys. Rev. B 85, 115309 (2012).

[28] D. P. S. McCutcheon and A. Nazir, Phys. Rev. Lett. 110, 217401 (2013).

[29] A. Moelbjerg, P. Kaer, M. Lorke, and J. Mork, Phys. Rev. Lett. 108, 017401 (2012).

[30] To ensure that this corresponds to the temperature of the QD itself, we have performed COMSOL simulations from which we estimate that the excitation laser heats the sample by no more than $10^{-6} \mathrm{~K}$.

[31] C. Santori, M. Pelton, G. Solomon, Y. Dale, and Y. Yamamoto, Phys. Rev. Lett. 86, 1502 (2001).

[32] C. Cohen-Tannoudji, J. Dupont-Roc, and G. Grynberg, Atom-Photon Interactions: Basic Processes and Applications (Wiley-VCH, New York, 1998).

[33] H. D. Robinson and B. B. Goldberg, Phys. Rev. B 61, R5086 (2000).

[34] A. Berthelot, I. Favero, G. Cassabois, C. Voisin, C. Delalande, Ph. Roussignol, R. Ferreira, and J. M. Gérard, Nat. Phys. 2, 759 (2006).

[35] J. Houel, A. V. Kuhlmann, L. Greuter, F. Xue, M. Poggio, B. D. Gerardot, P. A. Dalgarno, A. Badolato, P. M. Petroff, A. Ludwig, D. Reuter, A. D. Wieck, and R. J. Warburton, Phys. Rev. Lett. 108, 107401 (2012).

[36] J. P. Reithmaier, G. Sęk, A. Löffler, C. Hofmann, S. Kuhn, S. Reitzenstein, L. V. Keldysh, V. D. Kulakovskii, T. L. Reinecke, and A. Forchel, Nature (London) 432, 197 (2004); K. Hennessy, A. Badolato, M. Winger, D. Gerace, M. Atatüre, S. Gulde, S. Fält, E. L. Hu, and A. Imamoğlu, Nature (London) 445, 896 (2007).

[37] D. M. Toyli, C. F. de las Casas, D. J. Christle, V. V. Dobrovitski, and D.D. Awschalom, Proc. Natl. Acad. Sci. U.S.A. 110, 8417 (2013).

[38] G. Wrigge, I. Gerhardt, J. Hwang, G. Zumofen, and V. Sandoghdar, Nat. Phys. 4, 60 (2008).

[39] O. Astafiev, A. M. Zagoskin, A. A. Abdumalikov, Yu. A. Pashkin, T. Yamamoto, K. Inomata, Y. Nakamura, and J. S. Tsai, Science 327, 840 (2010).

[40] A. Batalov, C. Zierl, T. Gaebel, P. Neumann, I.-Y. Chan, G. Balasubramanian, P. R. Hemmer, F. Jelezko, and J. Wrachtrup, Phys. Rev. Lett. 100, 077401 (2008). 